\title{
Short-term changes of human acellular dermal matrix (Megaderm) in a mouse model
}

\author{
Yang Seok Kim, \\ Young Cheon Na, \\ Hyun Sik Yoon, \\ Woo Hoe Huh, \\ Ji Min Kim
}

Department of Plastic and Reconstructive Surgery, Wonkwang University Hospital, Iksan, Korea

\begin{abstract}
Background: Physicians tend to overcorrect when applying the acellular dermal matrix for reconstructive option because of volume decrement problem after absorption comparing with initial volume. However, there are no studies on the exact volume decrement and absorption rate with commercial products in South Korea. To figure out absorption rate of acellular dermal matrix product in South Korea (Megaderm), authors designed this experiment.

Methods: Nine mice were used and randomly divided into three groups by the time with sacrificing. The implant (Megaderm) was tailored to fit a cuboid form $(1.0 \mathrm{~cm} \times 1.0 \mathrm{~cm}$ in length and width and $2.0 \mathrm{~mm}$ in thickness). A skin incision was made at anterior chest with blade \#15 scalpel with exposing the pectoralis major muscle. As hydrated Megaderm was located upon the pectoralis major muscle, the skin was sutured with Ethilon \#5-0. After the surgical procedure, each animal group was sacrificed at 4, 8, and 12 weeks, respectively, for biopsies and histological analysis of the implants. All samples were stained with routine hematoxylin and eosin staining and Masson's trichrome staining and the thickness were measured. A measurements were analyzed using Friedman test. Statistically, the correlation between thicknesses of Megaderm before and after implantation was analyzed.

Results: After sacrificing the animal groups at postoperative 4, 8, 12 weeks, the mean tissue thickness values were $2.10 \pm 1.03 \mathrm{~mm}, 2.17 \pm 0.21 \mathrm{~mm}$, and $2.40 \pm 0.20 \mathrm{~mm}(p=0.368)$, respectively. The remaining ratios after absorption comparing with after initial hydrated Megaderm were $82.7 \%, 85.4 \%$, and $94.5 \%$, respectively. In histopathological findings, neovascularization and density of collagenous fiber was increased with time.

Conclusion: Author's hypothesis was absorption rate of implant would be increased over time. But in this experiment, there is no statistical significance between mean absorption thickness of implant and the time $(p=0.368)$. Also it can be affected by graft site, blood supply, and animals that were used in the experiment.
\end{abstract}

Keywords: Acellular dermal matrix / Alloderm / Megaderm / Surederm

\section{INTRODUCTION}

Using an autologous tissue is an ideal option to cover a soft tissue defects due to various reasons, particularly trauma, congen-

\section{Correspondence: Young Cheon Na}

Department of Plastic and Reconstructive Surgery, Wonkwang University Hospital, 895 Muwang-ro, Iksan 54538, Korea

E-mail: ycnadr@hanmail.net

Received October 8, 2018 / Revised December 4, 2018 / Accepted December 17, 2018 ital anomalies, or infections. However, covering with autologous tissue alone is limited because of its donor site morbidities and the lack of substantial quantities to harvest. Therefore, it is artificial dermal substitutes are also considered [1].

Cultured epidermal autograft, porous collagen and glycosaminoglycan matrix, and polyglycolic acid meshes were invented to cover the full-thickness skin loss by infection, burn wounds, frostbite, and trauma. However, the limitation of these options 
Table 1. The mean percentage volume persistence of Acellular dermal matrix in various study

\begin{tabular}{lc}
\hline Study & Mean volume persistence at 12 weeks (\%) \\
\hline Kim et al. [4] & $84.73 \pm 16.95$ \\
Hong et al. [5] & $80.39 \pm 8.21$ \\
Park et al. [6] & 74 \\
Sclafani et al. [7] & 48.3 \\
\hline
\end{tabular}

Values are presented as mean \pm standard deviation.

is that they are unstable and can induce immune reactions. To address this problem, allogenic acellular human dermis was invented, and reports are available demonstrating that it is stable and shows no immune reactions [2].

After an appropriate solvent and detergent process to eliminate cellular components, the next step is the freezing-drying process to obtain these acellular dermal matrix grafts (i.e., Megaderm) from human cadaver skin, which includes extracellular matrix and basement membrane complex and involves the removal of cellular components and epidermis [3].

In addition, it is also known that, when acellular dermal matrix is applied to the human body, it should be overcorrected because of the decrement of the initial volume by the tendency of absorption itself (Table 1). However, previous research on the exact reduced volumes of acellular dermal matrix and the corresponding absorption rates with time is scarce. Therefore, in the present study, we designed an experiment to find out the absorption rates of human acellular dermal matrix (Megaderm; L\&C BIO Inc., Seongnam, Korea) which is now on sale in South Korea.

\section{METHODS}

\section{Materials}

Nine mice were used (male and female; weight, $30 \pm 5 \mathrm{~g}$ ). The mice were kept under routine laboratory conditions with unrestricted access to food and water. The animals were kept in separate cages. Thereafter, the mice were randomly divided into three groups. Each group had been sacrificed at postoperative 4 weeks, 8 weeks, 12 weeks, respectively, to figure out implant (acellular dermal matrix) absorption rates. Megaderm (L\&C BIO Inc.) was used in all groups of nine animals.

\section{Surgical procedure}

Mice ( $n=9)$ were anesthetized using the Sevoflurane (Baxter International Inc., Deerfield, IL, USA). After shaving the anterior chest of animals, routine surgical preparation was performed with 10\% Potadine iodine solution. A skin incision of about $1.5 \mathrm{~cm}$ was made with blade \#15 scalpel with exposing the Pectoralis major muscle with dissected with a blunt scissor. As hydrated Megaderm was located upon the pectoralis major muscle, the skin was sutured with Ethilon \#5-0 without subcutaneous suture. Postoperative dressing was performed with Potadine iodine solution and Fucidin (Leo Pharma, Ballerup, Denmark) ointment. The operation site was opened to observe any infection signs. A parenteral antibiotics was not administered for preventing postoperative infections. After the surgical procedure, each animal group was sacrificed at 4 weeks, 8 weeks, and 12 weeks, respectively, for biopsies and histological analysis of the implants. All samples were stained with routine hematoxylin and eosin staining to measure the thickness of implants; Masson's trichrome staining was used to evaluate collagenous changes of implants.

\section{Measurement of thickness}

The implant (Megaderm) was not applied a unit of the whole product for each mice but was divided and tailored to fit a cuboid form $(1.0 \mathrm{~cm} \times 1.0 \mathrm{~cm}$ in length and width and $2.0 \mathrm{~mm}$ in thickness). Before hydration, the implants (Megaderm) were measured regarding their length, width, and thickness for 4 spots (12, 3, 6, and 9 oclock) with a caliper ruler. For hydration, the implants (Megaderm) were incubated in normal saline solution at room temperature for 10 minutes. After hydration, the implants were measured again as described above and the average thickness was computed.

\section{Statistical analysis}

For each group, the thicknesses of Megaderm before and after implantation with time were determined as mean and standard deviation. The Friedman test was used to figure out whether significant differences were present between the groups. The statistical analysis program IBM SPSS ver. 22.0 (IBM Corp., Armonk, NY, USA) was used. A $p$-value of $<0.05$ was considered to be statistically significant.

\section{RESULTS}

\section{General findings}

Two weeks after the surgical procedure, all groups showed the signs of swelling. However, no signs of infection or wound dehiscence were observed. Overall, there were no apparent differences among the three groups. Thin capsular formation was detected around the implant (Megaderm) after biopsies. However, it was easily separated by blunt dissection.

\section{Changes of tissue thickness}

Before hydration, the mean tissue thickness of Megaderm mea- 
sured with four spots was $2.28 \mathrm{~mm}$. However, after hydration, the mean tissue thickness of Megaderm measured with four spots was $2.54 \mathrm{~mm}$. The increment of thickness was $0.26 \mathrm{~mm}$ after hydration procedure.

After sacrificing the animal groups at postoperative 4, 8, 12 weeks, the mean tissue thickness values were $2.10 \pm 1.03 \mathrm{~mm}$, $2.17 \pm 0.21 \mathrm{~mm}$, and $2.40 \pm 0.20 \mathrm{~mm}(p=0.368)$, respectively. The remaining ratios after absorption comparing with after initial hydrated Megaderm were $82.7 \%, 85.4 \%$, and $94.5 \%$, respectively (Table 2).

\section{Histopathologic findings}

\section{At 4 weeks after operation}

A grafted Megaderm showed newly developed cell layer formation and small vessel formation. Minor inflammatory cells and fibroblasts were observed. The results of Masson's trichrome staining showed a lower density of collagenous fibers (Fig. 1).

\section{At 8 weeks after operation}

There were more developed fibroblast and vessel formations with defined capsular formation around a grafted implant. The results of Masson's trichrome staining showed a high density of collagenous fibers (Fig. 2).

\section{At 12 weeks after operation}

The results of Masson's trichrome showed an extremely high density of collagenous fibers that looked like a normal dermis tissue (Fig. 3). A borderline form between epithelium and broad fibrocytes was observed around a grafted implant. Comparing with 4 weeks' specimen, the structure of Megaderm itself was maintained. Diffuse infiltrations of collagenous fibers seemed to be apparent. Also, there was no histological difference between surface of fascial attached and dermal attached of the extracted implants.

Table 2. Change of mean tissue thickness with time

\begin{tabular}{|c|c|c|c|c|}
\hline Variable & 4 wk & $8 w k$ & 12 wk & $p$-value ${ }^{*}$ \\
\hline Thickenss (mm) & $2.10 \pm 1.03(82.7)$ & $2.17 \pm 0.21(85.4)$ & $2.40 \pm 0.20(94.5)$ & 0.368 \\
\hline
\end{tabular}

Values are presented as mean \pm standard deviation and the values in parenthesis are percentage of mean tissue thickness compared to hydrated Megaderm. ${ }^{*} p>0.05$.
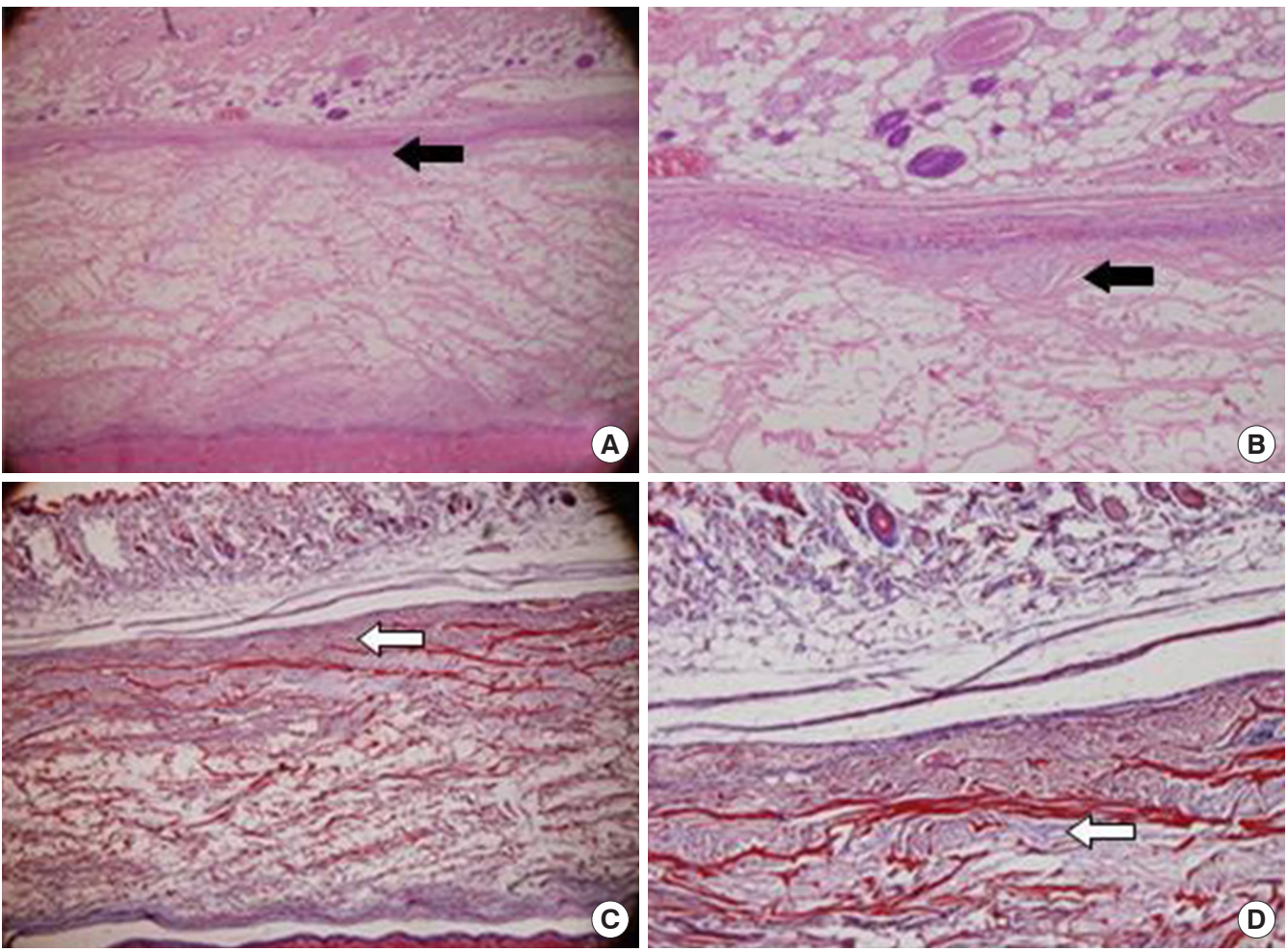

Fig. 1. Representative photographs of grafted human acellular dermal graft (Megaderm) at 4 weeks. (A, B) A grafted acellular cadaveric dermal graft showed newly developed cell layer formation and small vessels (black arrows; H\&E). (C, D) Lower density of collagenous fibers with Masson's trichrome staining (white arrows). (A, C) $\times 40$, (B, D) $\times 100$. 

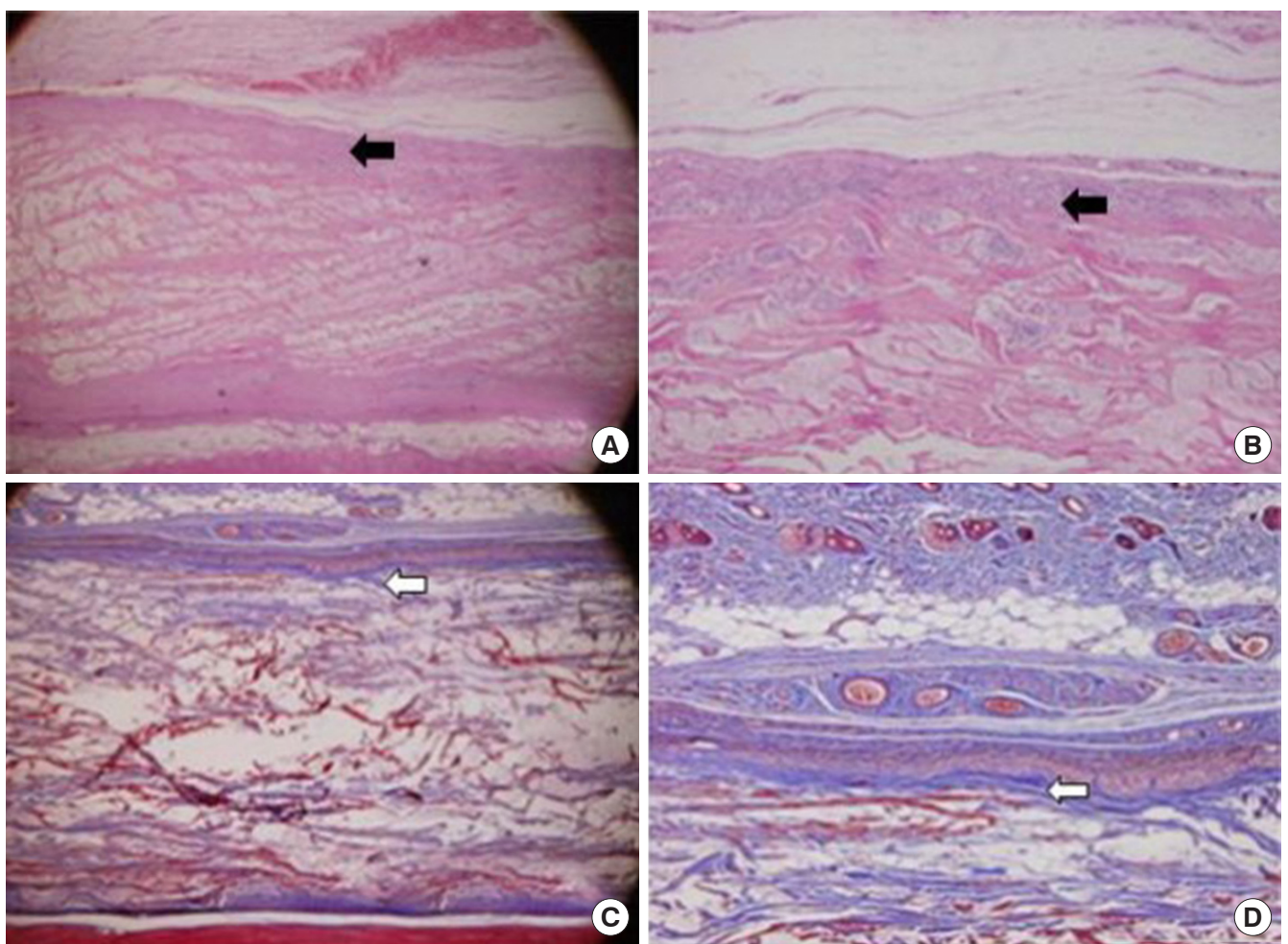

Fig. 2. Representative photographs of grafted human acellular dermal graft (Megaderm) at 8 weeks. (A, B) A grafted acellular cadaveric dermal graft showed much more fibrocyte like cells and small vessels (black arrows; H\&E). (C, D) High density of collagenous fibers with Masson's trichrome staining (white arrows). (A, C) $\times 40,(B, D) \times 100$.
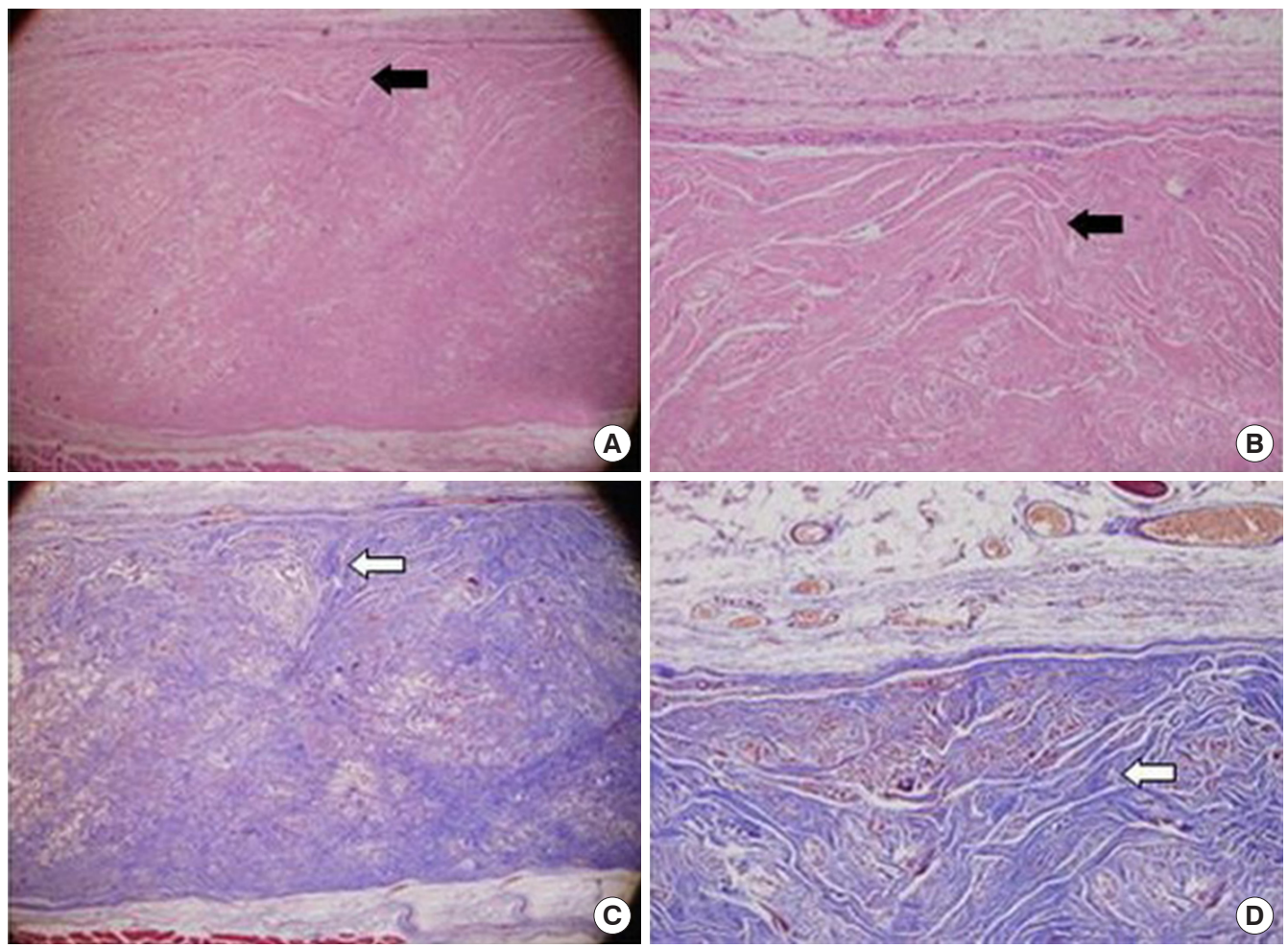

Fig. 3. Representative photographs of grafted human acellular dermal graft (Megaderm) at 12 weeks. (A, B) A grafted acellular cadaveric dermal graft showed diffused fibrocyte like cells and borderline form between epithelium (black arrows; H\&E). (C, D) Extremely high density of collagenous fibers look like a normal dermis tissue with Masson's trichrome staining (white arrows). (A, C) $\times 40,(B, D) \times 100$. 


\section{DISCUSSION}

The dermis layer consists of collagen fiber and extracellular matrix. It contributes to stability and elasticity of the skin layer. For centuries, there have been numerous attempts to hide soft tissue defects caused by numerous factors, particularly trauma or congenital anomalies. Full-thickness and split thickness skin grafts were workhorse for these attempts. Nicolle et al [8]. used the autologous dermis graft for facial wrinkle correction. However, covering the defects with the autologous tissue alone has limitations, due to its donor site morbidity and lack of its quantities to harvest. Donor site morbidity and lack of sufficient quantities to harvest are the limitations of using autologous tissue coverage. In addition to autologous tissue, various dermal substitutes were invented to cover the full-thickness skin loss by infection, burn wounds, frostbite, and trauma. However, the limitation of these options is that they are unstable and can induce immune reactions. To address this problem, allogenic acellular human dermis was invented, and reports are available demonstrating that it is stable and shows no immune reactions [2]. In 1995, Wainwright [9] was the first describe an acellular dermal matrix for the reconstruction of full-thickness burn wound with a split-thickness skin graft. The author noted that there were no immune reactions and a good cosmetic and functional result was obtained. Afterwards, an acellular dermal matrix has been widely used for breast reconstruction, contour irregularity corrections, and hernia repair. In cosmetic surgery, it is used for augmentation rhinoplasty, nasal irregularity correction, and secondary rhinoplasty [10-13].

Compared to conventional autologous full- or split-thickness skin grafts, a split thickness skin graft with acellular dermal matrix is reported to yield better cosmetic and functional results, as it can reduce the donor and graft site scar formation [14-16]. However, the complications of acellular dermal matrix include cellulitis, implant failure, seroma formation, wound dehiscence, and wound infection [17]. For the diverse usage of acellular dermal matrix, Flex HD (Ethicon), DermaMatrix (Synthes), AlloMax (Bard Davol), and SurgiMend (TEI Biosciences) were invented following Alloderm (LifeCell Corp.)

In previous studies, cosmetic and functional superiority of acellular dermal matrix was consistently demonstrated in previous research. However, most of these studies used Alloderm (LifeCell Corp.), so that research on acellular dermal matrix except for Alloderm (LifeCell Corp.) remains scarce.

Physicians tend to overcorrect when applying the acellular dermal matrix for reconstructive option because of volume decrement problem after absorption comparing with initial volume. However, there are no studies on the exact volume decrement and absorption rate with commercial products in South Korea. Therefore, in the present study, we designed an experiment to find out the exact absorption rate and volume decrement [4].

Wainwright [9] and Wainwright et al. [18] reported that fibroblast development and neovascularization started in acellular dermal matrix at 7 days after implantation. In 14 days after implantation, engraftment was made at the basement membrane complex with infiltration of fibroblast and the in-setting procedure.

Furthermore, Lee et al. [19] reported that implanted acellular dermal matrix was totally absorbed at 4 weeks after graft implantation. However, in general clinical practice, acellular dermal matrix of the thickness above $1 \mathrm{~mm}$ is usually used. However, the results of this experiment at 4 weeks after operation were far from total graft take. We assumed that difference of the result was contributed to the implant thickness.

In addition, Kim et al. [4] reported that the thickness of implanted acellular dermal matrix is inversely proportional with the absorption time by neovascularization, collagenous fiber formation, and replacement of autologous tissue. It was also observed that collagenous fiber infiltration was highly active and replaced implanted dermal matrixes at 8 weeks after implantation.

In a study of the relation of multi-layered acellular dermal matrix between absorption, Lee et al. [20] reported that longlasting thickness is proportional with the multiple layer structure of acellular dermal matrix. Considering the results of the two studies overviewed above, we concluded that it is variable the absorption time with depending on the thickness of implants. In this experiment, absorption thickness of Megaderm decreased and there is no statistically significance with time ( $p=0.368)$. This result is opposite to the general assumption that absorption rate of implant will increase with time. We assume that, with the flow of time, collagenous fiber became more infiltrative and conjunctive, and neovascularization was made with the framework of Megaderm as time goes by.

$\mathrm{Na}$ et al. [21] reported that dermal framework of human acellular dermal matrix was maintained throughout a long period after implantation by histological analysis using optical and electron microscope. This study would be the basis of authors' assumption. Gryskiewicz [22] reported that there was no contour change of nasal dorsum for the dorsal graft with acellular dermal matrix in dorsal irregularity correction at 1-year followup study. Lee and Koh [23] investigated the difference of acellular dermal matrix between temporoparietal fascia in the correction of the secondary cleft lip deformity. The results of this study showed that there was no statically significance between two groups with respect to volume absorption rate and autolo- 
gous tissue and acellular dermal matrix. Park et al. [6] announced that volume of Alloderm with implanted in nude mouse was steadily decreased at 12 weeks after graft. But later than 12 weeks after graft, the authors observed no volume decrement and maintained long period.

However, in a study of Alloderm graft at the postauricular area in adults, Sclafani et al. [7] described that only $20.2 \%$ of initial volume of Alloderm was remained after 1-year followup. Owens and Yukna [24] reported that most implanted Alloderm was totally absorbed at 4 months after implantation of the Alloderm with oral mucosa of dog. In histological analysis, differentiation between grafted Alloderm and around the normal tissue was difficult to find. Several authors reported that a high absorption rate was observed in lip augmentation, unlike the dorsal graft in nose contour irregularity correction, and assumed the cause was the lip motion. In this study, it is suggested that the wound bed of the bone or cartilage showed the lowest absorption of Alloderm [25].

In this experiment, the implant insertion plane was superior surface of Pectoralis major muscle. A motional plane is the distinguishing point comparing with previous study. Authors choose this plane in order to diversify the insertion plane. By doing so, authors had wanted to know a multifarious results about short-term changes of acellular dermal matrix implantation. Because most of studies were designed with an insertion plane for immobile or easily checked up site, the result of supramuscular plane could be a novelty. These conflicting results can be related to the fact that the absorption of acellular dermal matrix could be affected by graft site, blood supply, and animals that were used in the experiment.

Because this experiment was done with mouse study only not in human and only 12 weeks follow-up, general application to human is difficult with some limitations. Also if implant insertion plane is applied with difference, it could be contributed to absorption rate. Clinically, insertion plane of implants varies from subdermis to fascia. However, in the present study, the implant was placed only on pectoralis major muscle. In our experiment, the mean absorption thickness was 5.5\%. Measured with a ruler, the mean absorption caliper was $0.14 \mathrm{~mm}$. We measured a thickness of implant in place of measuring a volume of implant because a carved implant was cuboidal form and length and width of extracted implant was constant. The only altered figure was thickness. In conclusion, there is no statistical significance between the absorption rate and the time $(p=0.368)$. In clinical usage, overcorrection was not a prior consideration, because mean absorption of Megaderm was only $0.14 \mathrm{~mm}$ caliper. Additionally, changing an insertion plane or insertion site and following a longer period of thickness measurement will provide spe- cific and accurate information for clinicians interested in reconstruction with acellular dermal matrix.

\section{NOTES}

\section{Conflict of interest}

No potential conflict of interest relevant to this article was reported.

\section{Acknowledgments}

This study was supported by Wonkwang University in 2017.

\section{Ethical approval}

Animal testing was conducted in accordance with the ethical standards of Institutional Animal Experimentation Ethics Committee of Wonkwang University (No. WKU15-88).

\section{ORCID}

Yang Seok Kim https://orcid.org/0000-0003-3161-7284

Young Cheon Na https://orcid.org/0000-0003-3136-0351

Hyun Sik Yoon https://orcid.org/0000-0002-2294-5465

Woo Hoe Huh https://orcid.org/0000-0002-1379-7122

Ji Min Kim https://orcid.org/0000-0002-9819-0775

\section{REFERENCES}

1. Lew DH, Kim SM, Ahn SJ, Park BY. Histomorphometric study of tissue reaction of Gore-tex implant in the rat model. J Korean Soc Plast Reconstr Surg 2000;27:247-52.

2. Medalie DA, Eming SA, Tompkins RG, Yarmush ML, Krueger GG, Morgan JR. Evaluation of human skin reconstituted from composite grafts of cultured keratinocytes and human acellular dermis transplanted to athymic mice. J Invest Dermatol 1996; 107:121-7.

3. Bottino MC, Jose MV, Thomas V, Dean DR, Janowski GM. Freeze-dried acellular dermal matrix graft: effects of rehydration on physical, chemical, and mechanical properties. Dent Mater 2009;25:1109-15.

4. Kim HT, Ahn ST, Park JG. Absorption Rates of various-thickness human acellular dermal grafts(SureDerm (R)). J Korean Soc Plast Reconstr Surg 2003;30:224-30.

5. Hong JS, Kim WS, Yu YI, Kim HK. Comparison of survival of SureDerm(R), Permacol(R) graft in mouse. J Korean Soc Plast Reconstr Surg 2007;34:685-90.

6. Park DS, Kim HK, Kim SH, Kim SH, Lee TJ. Comparison of survival of human dermis, AlloDerm and Terudermis graft in nude mous. J Korean Soc Plast Reconstr Surg 2002;29:188-97.

7. Sclafani AP, Romo T 3rd, Jacono AA, McCormick SA, Cocker 
R, Parker A. Evaluation of acellular dermal graft (AlloDerm) sheet for soft tissue augmentation: a 1-year follow-up of clinical observations and histological findings. Arch Facial Plast Surg 2001;3:101-3.

8. Nicolle FV, Matti BA, Scamp T. Dermal and facial autografts in facial aesthetic surgery. Aesthetic Plast Surg 1992;16:219-25.

9. Wainwright DJ. Use of an acellular allograft dermal matrix (AlloDerm) in the management of full-thickness burns. Burns 1995;21:243-8.

10. Salzberg CA, Ashikari AY, Koch RM, Chabner-Thompson E. An 8-year experience of direct-to-implant immediate breast reconstruction using human acellular dermal matrix (AlloDerm). Plast Reconstr Surg 2011;127:514-24.

11. Breuing $\mathrm{KH}$, Warren SM. Immediate bilateral breast reconstruction with implants and inferolateral AlloDerm slings. Ann Plast Surg 2005;55:232-9.

12. Gamboa-Bobadilla GM. Implant breast reconstruction using acellular dermal matrix. Ann Plast Surg 2006;56:22-5.

13. Sherris DA, Oriel BS. Human acellular dermal matrix grafts for rhinoplasty. Aesthet Surg J 2011;31(7 Suppl):95S-100S.

14. Askari M, Cohen MJ, Grossman PH, Kulber DA. The use of acellular dermal matrix in release of burn contracture scars in the hand. Plast Reconstr Surg 2011;127:1593-9.

15. Gore DC. Utility of acellular allograft dermis in the care of elderly burn patients. J Surg Res 2005;125:37-41.

16. Rennekampff HO, Pfau M, Schaller HE. Acellular allograft dermal matrix: immediate or delayed epidermal coverage?
Burns 2002;28:100-1.

17. Adetayo OA, Salcedo SE, Bahjri K, Gupta SC. A meta-analysis of outcomes using acellular dermal matrix in breast and abdominal wall reconstructions: event rates and risk factors predictive of complications. Ann Plast Surg 2016;77:e31-8.

18. Wainwright D, Madden M, Luterman A, Hunt J, Monafo W, Heimbach D, et al. Clinical evaluation of an acellular allograft dermal matrix in full-thickness burns. J Burn Care Rehabil 1996;17:124-36.

19. Lee DH, Han DW, Baik BS. Histologic changes of various artificial dermis graft in rabbit. J Korean Soc Plast Reconstr Surg 2000;27:550-61.

20. Lee DW, Lee MC, Roh H, Lee WJ. Multilayered implantation using acellular dermal matrix into nude mice. J Mater Sci Mater Med 2014;25:2669-76.

21. Na YC, Kang YS, Ryu BS, Yang JY. Recontruction of soft tissue defect of feet and legs using Alloderm. J Korean Soc Plast Reconstr Surg 1999;26:996-1000.

22. Gryskiewicz JM. Dorsal Augmentation with AlloDerm. Semin Plast Surg 2008;22:90-103.

23. Lee KN, Koh KS. Acellular human dermis, a good option for correcting the free border deficiency in secondary cleft lip deformity. J Plast Reconstr Aesthet Surg 2012;65:356-61.

24. Owens KW, Yukna RA. Collagen membrane resorption in dogs: a comparative study. Implant Dent 2001;10:49-58.

25. Tobin HA, Karas ND. Lip augmentation using an Alloderm graft. J Oral Maxillofac Surg 1998;56:722-7. 